J. Dairy Sci. 98:4241-4241

http://dx.doi.org/10.3168/jds.2015-98-6-4241

(c) American Dairy Science Association ${ }^{\circledR}, 2015$.

\title{
Corrigendum to "Multilocus sequence typing of a dairy-associated Leuconostoc mesenteroides population reveals clonal structure with intragenic homologous recombination" (J. Dairy Sci. 98:2284-2293)
}

Wenyi Zhang, Wenjun Liu, Yuqing Song, Haiyan Xu, Bilige Menghe, Heping Zhang, and Zhihong Sun

The primer sequences for $m u r C$ and $m u r E$ in Table 1 (page 2285) were incorrect. The correct sequences $\left(5^{\prime}-3^{\prime}\right)$ are as follows:

\author{
murC_primerF: CCATTAGAGGCAGCAGGTA and \\ murC_primerR: GGTTTCAAAGAACGCAAGT \\ murE_primerF: GCCTTCTTGAATATCTCCC and \\ murE_primerR: CAGCGGTTAAATAAACTACATC
}

The authors regret the error.

\section{REFERENCES}

Zhang, W., W. Liu, Y. Song, H. Xu, B. Menghe, H. Zhang, and Z. Sun. 2015. Multilocus sequence typing of a dairy-associated Leuconostoc mesenteroides population reveals clonal structure with intragenic homologous recombination. J. Dairy Sci. 98(4):2284-2293. 